\title{
Minimum Number of Probes for Brain Dynamics Observability
}

\author{
Sérgio Pequito * Paul Bogdan ${ }^{\star}$ George J. Pappas *
}

\begin{abstract}
In this paper, we address the problem of placing sensor probes in the brain such that the system dynamics' are generically observable. The system dynamics whose states can encode for instance the fire-rating of the neurons or their ensemble following a neural-topological (structural) approach, and the sensors are assumed to be dedicated, i.e., can only measure a state at each time. Even though the mathematical description of brain dynamics is (yet) to be discovered, we build on its observed fractal characteristics and assume that the model of the brain activity satisfies fractional-order dynamics.

Although the sensor placement explored in this paper is particularly considering the observability of brain dynamics, the proposed methodology applies to any fractional-order linear system. Thus, the main contribution of this paper is to show how to place the minimum number of dedicated sensors, i.e., sensors measuring only a state variable, to ensure generic observability in discrete-time fractional-order systems for a specified finite interval of time. Finally, an illustrative example of the main results is provided using electroencephalogram (EEG) data.
\end{abstract}

\section{INTRODUCTION}

In the recent years, control theoreticians have resorted to the tools available to study, analyze and design dynamical systems to better understand the dynamical behavior of the brain [1], [2], [3], [4], [5], [6], [7]. Often, the dynamic model of the brain assumes a behavior at the neuron level, that is then analyzed in interaction with different neurons to which it communicates with [3], [2]. A commonly used model for the neuron is the Hodgkin and Huxley, but several alternatives have been proposed. Most of the models have the following similarity: a neuron fires (i.e., beams a signal to neighboring neurons) if a certain threshold (or, gain) on its state (or stimulus) is reached. Nonetheless, the evolution of the state is believed to be non-linear in general, with a time-varying threshold. Therefore, several experimental efforts concluded that several regions in the brain exhibit specific properties. In particular, the nonlinear dynamics in certain regions can be sequentially approximated by linear time-invariant systems, leading to a linear time-varying systems that can then be restricted to a finite possible dynamics due to some plasticity and invariant properties of the brain regions. Alternatively, the response to a stimulus may exhibit a long-range memory behavior which may indicate the presence of a fractional-order dynamics; for instance, the vesicular-ocular system,

This work was supported in part by the TerraSwarm Research Center, one of six centers supported by the STARnet phase of the Focus Center Research Program (FCRP) a Semiconductor Research Corporation program sponsored by MARCO and DARPA. P.B. acknowledges the support by NSF 1453860 and 1331610 grants.

* Department of Electrical and Systems Engineering, School of Engineering and Applied Science, University of Pennsylvania

$\diamond$ USC Ming Hsieh Department of Electrical Engineering, Viterbi School of Engineering, University of Southern California 
the fly motion sensitive neuron and neocortical neurons [8], just to name a few. Nonetheless, several other dynamical systems are described by similar dynamics and sensing capabilities. For instance, fractional-order dynamics include artificial pancreas [9], on-chip traffic regulation [10], traffic dynamics across road arteries [11], electrical and thermal machines, transmission and acoustics [12], just to name a few.

Hereafter, instead of determining a specific model to the brain's dynamics given the neurons activity (or an ensemble of these) and their interactions, to which several studies have been conducted [13], [14], we assume that the brain dynamics can be described by fractional-order dynamics. Then, we aim to provide a methodology to determine the minimum placement of dedicated sensors, i.e., sensors that measure a single state of the system, ensuring that the system is generically observable; more precisely, by generically observable (also known as structurally observable - see Section III for formal definition), we mean that almost all parametrizations (that are unknown) for a given structure of interaction (that can be known or imposed by neural-topological analysis). The reason we focus in determining such placement in the context of brain observability dynamics is threefold: (i) some probes (sensors) are invasive, i.e., require surgery to deploy the probes in the brain, which may be life threatening; (ii) nowadays deployment of probes in the brain neglects entirely the brain dynamics; and (iii) data collected in these probes is commonly used to feedback into the system, therefore, a smaller collection of data leads to a faster response time with potential implications in neurodegenerative diseases such as Parkinson's and epilepsy [15].

To the best of the authors knowledge there are no systematic methods to do probe (sensor) placement in the brain. Instead, researchers have focused on collecting data from different regions of the brain and/or developing new sensors leveraging some of the new materials (some biodegradable that do not require the extraction after a period of time). On the other hand, if we consider dynamical systems, most sensor placement methodologies are either heuristic or greedy due to the combinatorial nature of the problem, see [16] and references therein. Structural systems theory studies the coupling between state variables rather than the specific strength of the coupling, suitable to the problems explored hereafter, since these are not available neither measurable directly, therefore they need to be estimated after data has been collected. The structural treatment of dynamical properties of switching systems and, in particular, the obtention of sufficient and necessary conditions to ensure structural observability, i.e., structural observable for some non-empty window of time, have been explored in [17], [18]. This approach contrasts with the one presented in [19], where conditions to ensure structural observability for all non-empty windows of time. In [19], the deployment of dedicated sensors was explored, but the sensor placement to ensure structural observability for some non-empty window of time, has not yet been addressed. The present work also extends [16], [20], where the minimum placement of dedicated sensors to ensure structural observability was addressed for linear time-invariant systems.

Main Contribution: The main contributions of the paper is to show how to place the minimum number of dedicated sensors to ensure structural observability in discrete-time fractional-order systems for a specified finite interval of time.

The rest of the this paper is organized as follows. First, in Section II we present the formal statements addressed 
in the present paper. We introduce and revise some preliminary concepts and results in Section III. In Section IV we present the main technical results, followed by an illustrative example in Section $\mathrm{V}$. Finally, conclusions and discussion avenues for further research are presented in Section VI.

\section{Problem Statement}

In this section, we formally introduce the dedicated sensor placement for discrete-time fractional order dynamics. Formally, this problem is described as follows.

$\mathcal{P}_{1}$ Consider a model dynamics described by a linear discrete-time fractional-order system as follows

$$
\Delta^{\alpha} x_{k+1}=\left[\begin{array}{c}
\Delta^{\alpha_{1}} x_{k+1}^{1} \\
\Delta^{\alpha_{2}} x_{k+1}^{2} \\
\vdots \\
\Delta^{\alpha_{n}} x_{k+1}^{n}
\end{array}\right]=\sum_{j=0}^{k+1} A_{j} x_{k}
$$

where $\alpha_{i} \in \mathbb{R}^{+}(i=1, \ldots, n), A_{0}=A$ and $A_{j}=\operatorname{diag}\left(-(-1)^{j+1}\left(\begin{array}{c}\alpha_{1} \\ j+1\end{array}\right),-(-1)^{j+1}\left(\begin{array}{c}\alpha_{2} \\ j+1\end{array}\right), \ldots,-(-1)^{j+1}\left(\begin{array}{c}\alpha_{n} \\ j+1\end{array}\right)\right)$, for brevity, we refer to the dynamics in $(1)$ as $\mathcal{F}(A ; \boldsymbol{\alpha}, K)$ which is associated with the representation of all matrices $A_{j}$, with $j=0, \ldots, K$. Please note that $\alpha$ is a non-integer number and the term $\left(\begin{array}{c}\alpha \\ j\end{array}\right)=\frac{\Gamma(\alpha+1)}{\Gamma(j+1) \Gamma(\alpha-j+1)}$ is expressed via the Gamma function $\Gamma(x)=\int_{0}^{\infty} t^{x-1} e^{-t} d t[21]$.

Hereafter, given $\mathcal{F}(A ; \boldsymbol{\alpha}, K)$ we aim to determine the minimum number of dedicated sensors $\mathcal{J}$ required to ensure that the linear discrete-time fractional-order system (1) with measured output

$$
y_{k}=\mathbb{I}_{n}^{\mathcal{J}} x_{k},
$$

is structurally observable, i.e.,

$$
\begin{array}{cc}
\arg \min _{\mathcal{J} \subset\{1, \ldots, n\}} & |\mathcal{J}| \\
\text { s.t. } & \left(\mathcal{F}(A ; \boldsymbol{\alpha}, K), \mathbb{I}_{n}^{\mathcal{J}}\right) \text { is structurally observable, }
\end{array}
$$

where $\mathcal{J} \subseteq\{1, \ldots, n\}$ denotes the set of indices of the dedicated sensors, $\mathbb{I}_{n}^{\mathcal{J}}$ represents the subset of columns of the identity matrix $\mathbb{I}_{n}$ with indices in $\mathcal{J}$, and $\left(\mathcal{F}(A ; \boldsymbol{\alpha}, K), \mathbb{I}_{n}^{\mathcal{J}}\right)$ describes the system (1)-(3).

Notice that given $K, K^{\prime}$ where $K^{\prime}>K$, we may have two different solutions to (1) with $\mathcal{F}(A ; \boldsymbol{\alpha}, K)$ and $\mathcal{F}\left(A ; \boldsymbol{\alpha}, K^{\prime}\right)$ for an arbitrary $k$.

\section{PRELIMINARIES AND TERMINOLOGY}

In this section, we review some notions of observability to discrete-time fractional-order systems, and their counterpart using structural systems theory [22].

We start by recalling that a solution in closed-form to (1) can be determined and given as follows.

Lemma 1 ([23], [12] ): The solution to (1) is given as follows:

$$
x_{k+1}=G_{k+1} x_{0}
$$


where

$$
G_{k}=\left\{\begin{array}{cl}
A & \text { for } k=0, \\
\sum_{j=0}^{k-1} A_{j} G_{k-1-j} & \text { for } k \geq 1 .
\end{array}\right.
$$

The notion of observability for discrete-time fractional-order systems is revisited in the next definition.

Definition 1: The fractional-order system modeled by (1) and (2) is observable at time $k=0$ if and only if there exists some $K>0$ such that the state $x_{0}$ at time $k=0$ can be uniquely determined from the knowledge of the measured output $y_{k}$ with $k=1, \ldots, K$.

Of a particular interest is the notion of the observability matrix that is related with the system's observability.

Definition 2 ([23], [|12] $)$ : The observability matrix associated with the discrete-time fractional order dynamics for a given time $k$ as described in (1), and the measured output

$$
y_{k}=C x_{k},
$$

is given as follows:

$$
\mathcal{O}_{k}^{f}=\left[\left(C G_{0}\right)^{\top}\left(C G_{1}\right)^{\top} \ldots\left(C G_{k-1}\right)^{\top}\right]^{\top}
$$

Additionally, we have the following result.

Theorem 1: The system described by (1)-(5) is observable if and only if there exists a finite time $K$ such that $\operatorname{rank}\left(\mathcal{O}_{K}^{f}\right)=n$.

Given that the precise numerical values of the network parameters are generally not available for the largescale systems of interest, a natural direction is to consider structured systems [24] based reformulations of the above topology design problems, which we pursue in this paper. Representative work in structured systems theory may be found in [25], [26], [27], [28], see also the survey [22] and references therein. The main idea is to reformulate and study an equivalent class of systems for which system-theoretic properties are investigated based on the location of zeroes/non-zeroes of the state space representation matrices. Properties such as observability, in this framework, referred as structural observability, initially formalized for linear time-invariant systems [25]. In fact, structural observability is a generic property, i.e., almost all (with respect to the Lebesgue measure) realizations satisfying a given structure are observable [27]. Given the similarity between observability criteria of the lineartime invariant and the discrete-time fractional order dynamics, one can readily extend the notion of structural observability to the latter. More precisely, a pair $(\mathcal{F}(A ; \boldsymbol{\alpha}, K), C)$ is said to be structurally observable if there exists a pair $\left(\mathcal{F}\left(A^{\prime} ; \boldsymbol{\alpha}, K\right), C^{\prime}\right)$ with the same structure as $(\mathcal{F}(A ; \boldsymbol{\alpha}, K), C)$, i.e., same locations of zeroes and non-zeroes, such that $\left(\mathcal{F}\left(A^{\prime} ; \boldsymbol{\alpha}, K\right), C^{\prime}\right)$ is observable. By density arguments [27], it may be shown that if a pair $(\mathcal{F}(A ; \boldsymbol{\alpha}, K), C)$ is structurally observable, then almost all (with respect to the Lebesgue measure) pairs with the 
same structure as $(\mathcal{F}(A ; \boldsymbol{\alpha}, K), C)$ are observable. In essence, structural observability is a property of the structure of the pair $(\mathcal{F}(A ; \boldsymbol{\alpha}, K), C)$ and not the specific numerical values.

Now, we associate with each subsystem a directed graph (digraph) $\mathcal{D} \equiv \mathcal{D}\left(A, \mathbb{I}_{n}^{\mathcal{J}}\right)=(\mathcal{V}, \mathcal{E})$, referred to as system digraph, with vertex set $\mathcal{V}$ and edge set $\mathcal{E}$, where $\mathcal{V}=\mathcal{X} \cup \mathcal{Y}$ with $\mathcal{X}=\left\{x^{1}, \ldots, x^{n}\right\}$ and $\mathcal{Y}=\left\{y^{1}, \ldots, y^{|\mathcal{J}|}\right\}$ represents the state and output vertices, respectively. In addition, $\mathcal{E}=\mathcal{E}_{\mathcal{X}, \mathcal{X}} \cup \mathcal{E}_{\mathcal{X}, \mathcal{Y}}$ where $\mathcal{E}_{\mathcal{X}, \mathcal{X}}=\left\{\left(x^{k}, x^{j}\right)\right.$ : $\left.A_{j k} \neq 0\right\}$ and $\mathcal{E}_{\mathcal{X}, \mathcal{Y}}=\left\{\left(x^{j}, y^{j}\right): j \in \mathcal{J}\right\}$ represents the state edges and output edges, respectively. Similarly, we can define a state digraph $\mathcal{D}(A)=\left(\mathcal{X}, \mathcal{E}_{\mathcal{X}, \mathcal{X}}\right)$. Later, the matrix $A$ is given in terms of $A=A_{1} \vee \ldots \vee A_{m}$, where $\vee$ corresponds to the entry-wise operation where if at least one of the entries is non-zero, then it provides a non-zero entry, and zero otherwise. A state vertex is said to be non-accessible by an output vertex if there exists no directed path, i.e., a sequence of directed edges where every edge ends in a vertex that is starting of another edges and no vertex is used twice, the state vertex to any output vertex. Additionally, we need to introduce the notion of a bipartite graph $\mathcal{B}(M)$ associated with a $m_{1} \times m_{2}$ matrix $M$ given by $\mathcal{B}(M)=\left(\mathcal{R}, \mathcal{C}, \mathcal{E}_{\mathcal{C}, \mathcal{R}}\right)$, where $\mathcal{R}=\left\{r_{1}, \ldots, r_{m_{1}}\right\}$ and $\mathcal{C}=\left\{c_{1}, \ldots, c_{m_{2}}\right\}$ correspond to the labeling row vertices and column vertices, respectively; further, $\mathcal{E}_{\mathcal{C}, \mathcal{R}}=\left\{\left(c_{j}, r_{i}\right): M_{i j} \neq 0\right\}$. The bipartite graph is an undirected graph with vertex set given by the union of the partition sets $\mathcal{C}$ and $\mathcal{R}$, which we refer to as left and right vertex sets, respectively. A matching $M \subset \mathcal{E}_{\mathcal{C}, \mathcal{R}}$ is a collection of edges that have no vertices in common. A maximum matching is a matching with maximum cardinality among all possible matchings. For ease of reference, if a vertex in the left and right vertex set does not belong to an edge in a maximum matching we refer to as a right- and left-unmatched vertex, respectively. In addition, we can consider weights associated with the edges in a bipartite graph, so we can consider the problem of determining the maximum matching with the minimum sum of the weights, that we refer to as the minimum weight maximum matching. A digraph $\mathcal{D}_{S}=\left(\mathcal{V}_{S}, \mathcal{E}_{S}\right)$ is a subgraph of $\mathcal{D}=(\mathcal{V}, \mathcal{E})$ if $\mathcal{V}_{S} \subseteq \mathcal{V}$ and $\mathcal{E}_{S} \subseteq \mathcal{E}$. Finally, a strongly connected component (SCC) is a maximal subgraph (there is no other subgraph, containing it, with the same property) $\mathcal{D}_{S}=\left(\mathcal{V}_{S}, \mathcal{E}_{S}\right)$ of $\mathcal{D}$ such that for every $u, v \in \mathcal{V}_{S}$ there exists a path from $u$ to $v$ and from $v$ to $u$. We can create a directed acyclic graph (DAG) by visualizing each SCC as a virtual node, where there is a directed edge between vertices belonging to two SCCs if and only if there exists a directed edge connecting the corresponding SCCs in the digraph $\mathcal{D}=(\mathcal{V}, \mathcal{E})$, the original digraph. The DAG associated with $\mathcal{D}(A)$ can be computed efficiently in $\mathcal{O}(|\mathcal{V}|+|\mathcal{E}|)[29]$. The SCCs in the DAG may be further categorized as follows.

Definition 3: [16] An SCC is said to be linked if it has at least one incoming/outgoing edge from another SCC. In particular, an SCC is non-bottom linked if it has no outgoing edges from its vertices to the vertices of another SCC.

Finally, consider a $m_{1} \times m_{2}$ matrix $M$, and let $\mathbb{M}=\left\{P \in \mathbb{R}^{m_{1} \times m_{2}}: P_{i j}=0\right.$ if $\left.M_{i j}=0\right\}$, then the generic rank (g-rank) of $M$ is given by $\mathrm{g}$-rank $(M)=\max _{P \in \mathbb{M}} \operatorname{rank}(P)$.

Now, we revise the structural observability necessary and sufficient conditions for linear switching systems.

Theorem 2 ([[18]): Consider a linear continuous-time switching system 


$$
\dot{x}(t)=A_{\sigma(t)} x(t)
$$

where $\sigma: \mathbb{R}^{+} \rightarrow M \equiv\{1, \ldots, m\}$ is a switching signal, and $x(t) \in \mathbb{R}^{n}$ the state of the system at the instance of time $t$. In addition, let the measured output to be given by

$$
y(t)=C x(t) .
$$

The linear continuous-time switching system (6)-(7) is structurally observable if and only if the following two conditions hold:

(i) $\mathcal{D}\left(A_{1} \vee \ldots \vee A_{m}, \mathbb{I}_{n}^{\mathcal{J}}\right)$ has no non-accessible state vertex;

(ii) $\operatorname{g-rank}\left(\left[A_{1}, \ldots, A_{m}, \mathbb{I}_{n}^{\mathcal{J}}\right]\right)=n$.

In addition, consider the following intermediate results.

Lemma 2 ([[16]): $\mathcal{D}\left(A_{1} \vee \ldots \vee A_{m}, \mathbb{I}_{n}^{\mathcal{J}}\right)$ has no non-accessible state vertex if and only if there exits an edge to an output vertex in $\mathcal{D}\left(A_{1} \vee \ldots \vee A_{m}, \mathbb{I}_{n}^{\mathcal{J}}\right)$ from a state vertex in each non-bottom linked SCC of the DAG associated with $\mathcal{D}\left(A_{1} \vee \ldots \vee A_{m}\right)$.

Lemma 3 ([24]): There exists a maximum matching of $\mathcal{B}(M)$ with size $n$ if and only if g-rank $(M)=n$. $\quad \diamond$

\section{MAin Results}

In this section, we present the main results of this paper. More precisely, we provide the solution to $\mathcal{P}_{1}$ that can be determined using Algorithm 1. Algorithm 1 is shown to be correct and with polynomial complexity in Theorem 4

First, we provide necessary and sufficient conditions to ensure structural observability of discrete time fractionalorder dynamical systems.

Theorem 3: A discrete time fractional-order dynamical system (1)-(2) is structurally observable at time $K$ if and only if the following two conditions hold:

(i) $\mathcal{D}\left(G_{0} \vee G_{1} \vee \ldots \vee G_{K}, \mathbb{I}_{n}^{\mathcal{J}}\right)$ has no non-accessible state vertex;

(ii) $\operatorname{g-rank}\left(\left[G_{0}, G_{1}, \ldots, G_{K}, \mathbb{I}_{n}^{\mathcal{J}}\right]\right)=n$,

where $G_{i}$ is as described in (4).

Proof: First, we notice that a linear continuous-time switching system (6)-(7) is structurally observable if the observability matrix associated with it given by

$$
\begin{gathered}
\mathcal{O}_{k}^{s}=\left[C^{\boldsymbol{\top}}\left(C A_{1}\right)^{\top} \ldots\left(C A_{m}\right)^{\top}\left(C A_{1} A_{2}\right)^{\top}\left(C A_{2} A_{1}\right)^{\top}\right. \\
\left.\ldots\left(C A_{\rho(1)}^{i_{1}} \ldots A_{\rho(m)}^{i_{m}}\right)^{\top} \ldots\left(C A_{1}^{n-1}\right)^{\top} \ldots\left(C A_{m}^{n-1}\right)^{\top}\right]^{\top},
\end{gathered}
$$

has generic rank equal to $n$. From an algebraic point of view, if we set $A_{\sigma(i)}=G_{i-1}$ (with $\left.i=1, \ldots, K+1\right)$ and $C=\mathbb{I}_{n}^{\mathcal{J}}$. Then, following the same steps as in Theorem 4 in [18], we obtain that the generic rank of $\mathcal{O}_{K+1}^{s}$ equals that of $\mathcal{O}_{K}^{f}$. Consequently, conditions (i)-(ii) follow by invoking Theorem 2. 
As a direct consequence of Theorem 3, by invoking Lemma 3 and Lemma 2, we obtain the following result.

Corollary 1: A fractional-order dynamical system (1)-(2) is structurally observable at time $K$ if and only if the following two conditions hold:

(i) there exits an edge to an output vertex in $\mathcal{D}\left(G_{0} \vee G_{1} \vee \ldots \vee G_{K}, \mathbb{I}_{n}^{\mathcal{J}}\right)$ from a state vertex in each non-bottom linked SCC of the DAG associated with $\mathcal{D}\left(G_{0} \vee G_{1} \vee \ldots \vee G_{K}\right)$;

(ii) there exists a maximum matching of $\mathcal{B}\left(\left[G_{0} \vee G_{1} \vee \ldots \vee G_{K}, \mathbb{I}_{n}^{\mathcal{J}}\right]\right)$ with size $n$, where $G_{i}$ is as described in (4).

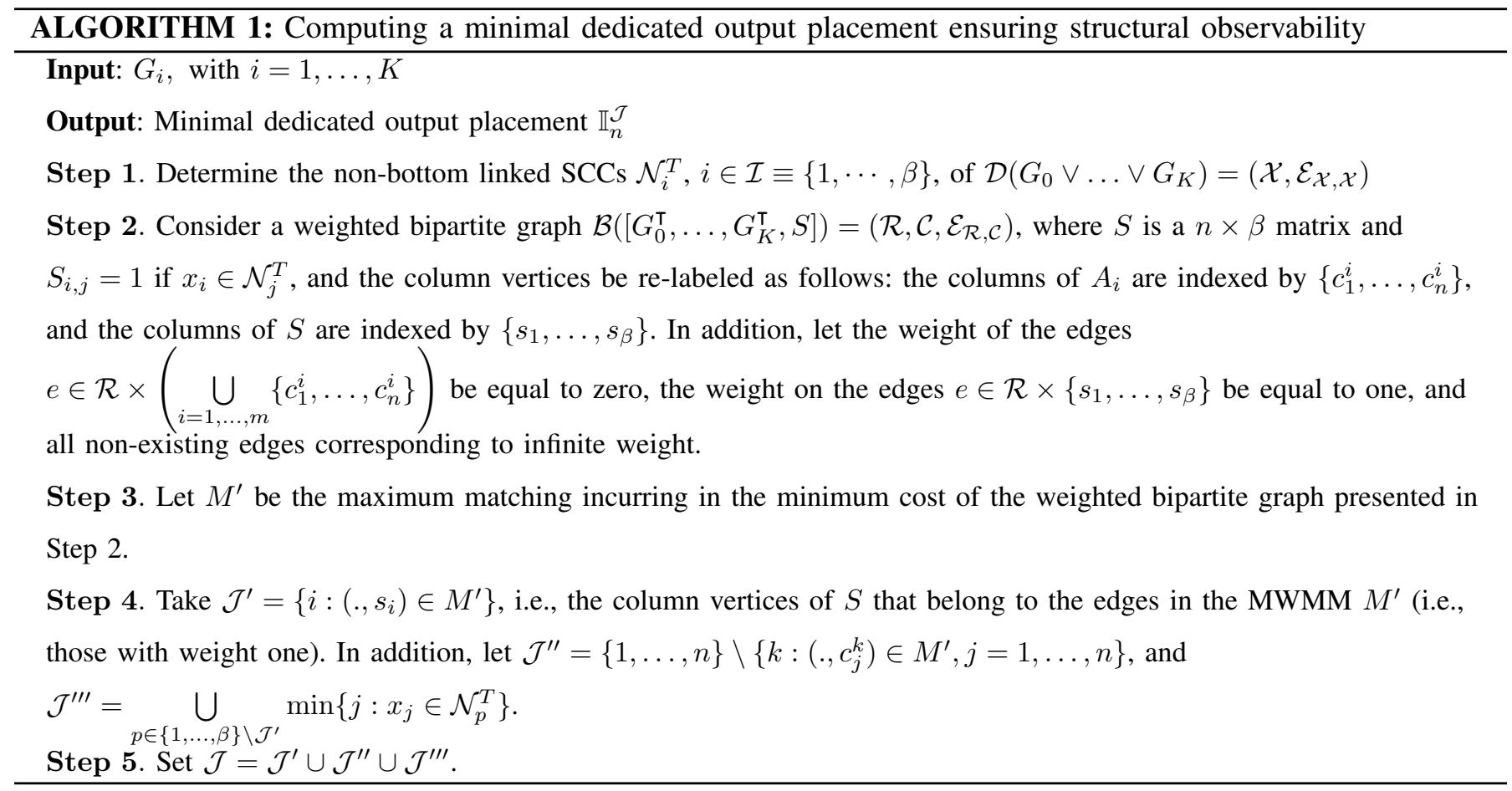

Now, we provide an efficient algorithmic procedure to compute a solution to $\mathcal{P}_{1}$, described in Algorithm 1 , that ensures that both conditions in Corollary 1 are satisfied. Briefly, Algorithm 1 consists in finding a minimum weight maximum matching (MWMM) of a bipartite graph $\mathcal{B}\left(\left[G_{0}^{\top}, \ldots, G_{K}^{\top}, S\right]\right)$, where the matrix $S$ has as many columns as the number of non-bottom linked SCCs in the DAG representation of $\mathcal{D}\left(G_{0} \vee \ldots \vee G_{K}\right)$, and the non-zero entries in column $i$ of $S$ correspond to the indices of the state variables that belong to the $i$-th non-bottom linked SCC. In addition, we consider weights in the edges of the bipartite graph: those associated with the nonzero entries of $A_{i}$ have zero weight, unitary weight is considered to the nonzero entries in $S$, and infinite weight otherwise.

Therefore, if an edge with unitary weight belongs to the MWMM, then it contains a row vertex, which implies that dedicated sensors need to be assigned to the state variable with the index of the row vertex, whereas all the remaining unitary edges not in the MWMM mean that these cannot be used to further increase the cardinality of the matching. Yet, notice that $\mathcal{B}\left(\left[G_{0}^{\top}, \ldots, G_{K}^{\top}, \mathbb{I}_{n}^{\mathcal{J}^{\prime}}\right]\right)$ may not have a maximum matching of size $n$; hence, additional dedicated sensors indexed by $\mathcal{J}^{\prime \prime}$ need to be considered, corresponding to the indices of the row vertices that are unmatched (i.e., the left-unmatched vertices of $\mathcal{B}\left(\left[G_{0}^{\top}, \ldots, G_{K}^{\top}, \mathbb{I}_{n}^{\mathcal{J}^{\prime}}\right]\right)$ associated with the MWMM). In summary, 
the indices in $\mathcal{J}^{\prime}$ correspond to the sensors that increase the g-rank and minimize the number of non-accessible state vertices, since they are assigned to non-bottom linked SCCs of $\mathcal{D}\left(G_{0} \vee \ldots \vee G_{K}\right)$, whereas $\mathcal{J}^{\prime \prime}$ ensures that the g-rank is equal to $n$. Thus, the last step consists in considering an additional subset of dedicated sensors $\mathcal{J}^{\prime \prime \prime}$ to ensure that there are no non-accessible state vertices in $\mathcal{D}\left(G_{0} \vee \ldots \vee G_{K}, \mathbb{I}_{n}^{\mathcal{J}}\right)$, where $\mathcal{J}=\mathcal{J}^{\prime} \cup \mathcal{J}^{\prime \prime} \cup \mathcal{J}^{\prime \prime \prime}$; in other words, $\mathcal{J}^{\prime \prime \prime}$ consists of indices of the minimum dedicated sensors assigned to a state variable in each non-bottom linked SCC that contains non-accessible state vertices.

The next result establishes the correctness and analyzes the implementation complexity of Algorithm 1.

Theorem 4: Algorithm 1 is correct, i.e., it provides a solution to $\mathcal{P}_{1}$. Furthermore, its computational complexity is $\mathcal{O}\left(|\mathcal{C}|^{3}\right)$, where $|\mathcal{C}|$ denotes the number of column vertices in $\mathcal{B}\left(\left[G_{0}^{\top}, \ldots, G_{K}^{\mathrm{\top}}, S\right]\right)$.

Proof: The correctness of Algorithm 1 follows from noticing that the indices in $\mathcal{J}^{\prime}$ identifies the minimum set of dedicated outputs that simultaneously maximizes the increase in the g-rank of $\left[G_{0}^{\top}, \ldots, G_{K}^{\top}, \mathbb{I}_{n}^{\mathcal{J}^{\prime}}\right]$ with respect to $\left[G_{0}^{\boldsymbol{\top}}, \ldots, G_{K}^{\boldsymbol{\top}}\right]$ by $\left|\mathcal{J}^{\prime}\right|$, and the dedicated outputs assigned to state variables in different non-bottom linked SCCs. This follows from observing that (by construction) $\mathcal{B}\left(\left[G_{0}^{\top}, \ldots, G_{K}^{\top}\right]\right)$ incurs in a minimum weight maximum matching $M$ with zero weight and size $|M|$. From Lemma 3, it follows that $\mathrm{g}$-rank $\left(\left[G_{0}^{\boldsymbol{\top}}, \ldots, G_{K}^{\mathrm{\top}}\right]\right)=|M|$. Subsequently, a minimum weight maximum matching $M^{\prime}$ of $\mathcal{B}\left(\left[G_{0}^{\top}, \ldots, G_{K}^{\top}, S\right]\right)$ equals $\left|M^{\prime}\right|-|M|$; hence, increasing by $\left|M^{\prime}\right|-|M|$ the g-rank of $\left[G_{0}^{\boldsymbol{\top}}, \ldots, G_{K}^{\boldsymbol{\top}}, \mathbb{I}_{n}^{\mathcal{J}^{\prime}}\right]$ with respect to $\left[G_{0}^{\boldsymbol{\top}}, \ldots, G_{K}^{\boldsymbol{\top}}\right]$, and contributing to satisfy condition (ii) in Corollary 1 - but may not be enough yet to ensure this condition, which is accounted for with the set $\mathcal{J}^{\prime \prime}$. In addition, by construction of $S$ it follows that $\mathbb{I}_{n}^{\mathcal{J}^{\prime}}$ correspond to dedicated outputs that are assigned to state variables in different non-bottom linked SCCs; hence, $\left|\mathcal{J}^{\prime}\right|$ non-bottom linked SCCs have outgoing edges from its state variables in different outputs in the system digraph; thus, contributing to satisfy condition (i) in Corollary 1 - but may not be enough yet to ensure this condition, which is accounted for with the set $\mathcal{J}^{\prime \prime \prime}$. Subsequently, the number of total number of additional dedicated outputs $\mathbb{I}_{n}^{\mathcal{J}^{\prime \prime}}$ required such that g-rank $\left(\left[G_{0}^{\boldsymbol{\top}}, \ldots, G_{K}^{\boldsymbol{\top}}, \mathbb{I}_{n}^{\mathcal{J}^{\prime}}, \mathbb{I}_{n}^{\mathcal{J}^{\prime \prime}}\right]\right)=n$ is minimized by considering Step 4. Similarly, the number of total number of additional dedicated outputs $\mathbb{I}_{n}^{\mathcal{J}^{\prime \prime \prime}}$ required such that there exist no non-accessible state vertices in $\mathcal{D}\left(G_{0} \vee \ldots \vee G_{K}, \mathbb{I}_{n}^{\mathcal{J}^{\prime} \cup \mathcal{J}^{\prime \prime \prime}}\right)$ is minimized by considering Step 4. Notice that $\mathbb{I}_{n}^{\mathcal{J}^{\prime \prime}}$ are not assigned to non-bottom linked SCCs, otherwise they would have been considered in $\mathbb{I}_{n}^{\mathcal{J}^{\prime}}$. Therefore, by setting $\mathcal{J}=\mathcal{J}^{\prime} \cup \mathcal{J}^{\prime \prime} \cup \mathcal{J}^{\prime \prime \prime}$, as in Step 5, we obtain a solution to $\mathcal{P}_{1}$, since Corollary 1$]$ yields.

The computational complexity follows from noticing that Step 2 can be solved using the Hungarian algorithm that finds a MWMM in $\mathcal{O}\left(\max \{|\mathcal{C}|,|\mathcal{R}|\}^{3}\right)$, whereas all other steps have linear complexity; hence, Step 2 dominates the final computational complexity, leading to the final complexity of $\mathcal{O}\left(|\mathcal{C}|^{3}\right)$ obtained, where we note that $|\mathcal{C}| \geq|\mathcal{R}|$.

\section{AN ILLUSTRATIVE EXAMPLE}

In what follows, we consider a brain activity dataset [30] describing the dynamics of the brain while the individuals perform different motor and imagery tasks. The brain activity was measured using the brain-computer interface and 
consists of 64-channel electroencephalogram (EEG). Each individual performed 14 experimental runs consisting of one minute with eyes open, one minute with eyes closed, and three two-minute runs of interacting sessions of opening/closing the corresponding left/right fist as a function of where the targets appear on screens. To this end, a geodesic sensor net for EEG was considered (illustrated in Figure 1), which sensor location schematics and enumeration is presented in Figure 2 .

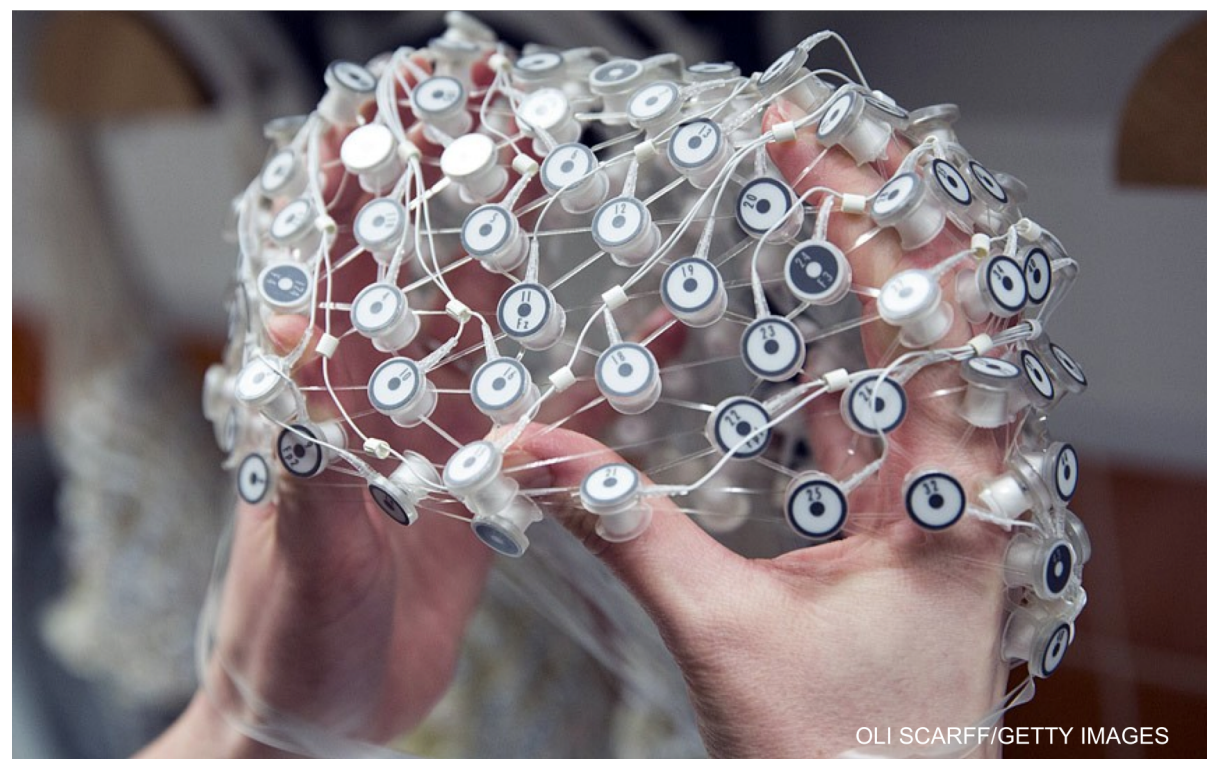

Fig. 1. 64-channel geodesic sensor net for an EEG.

Using the wavelet technique described in [31], we estimated the parameters $\alpha_{j}(j=1, \ldots, 64)$ in (1), corresponding to the fractional order derivatives governing the dynamics of the monitoring neuronal regions and the coupling matrix $A$ that encapsulates the spatial fractal connectivity among different brain regions. To give some intuition of how spread the parameters $\alpha_{j}$ corresponding to the fractional order derivatives are, the estimated values range between 0.97 and 1.28. Hence, providing evidence of the fractional order dynamics; more precisely, some brain regions exhibit pronounced long-range memory dynamics which can be better captured by fractional order state space representation. In Figure 3, we contrast the recorded and simulated data on channel 64 (depicted in blue in Figure 2), during the first two-minutes run of interacting sessions of opening/closing the corresponding left/right fist as a function of where the targets appear on screens.

A common approach in determining the brain connectivity is to consider the correlations between different measured signals [32]. In contrast, we advocate for a structural approach of the signal dynamics induced by the sparsification of the coupling matrix $A$. This structural analysis offers a robust mathematical framework for capturing both the spatial and temporal interactions among distant brain regions and identifying the minimum number of sensors that provide full observable description of brain dynamics. Following the theoretical results and analysis outlined in Corollary 2, by resorting to Algorithm 1, we determine the optimal number of sensors as a function of the sparsity present in the coupling matrix $A$ for a time horizon equal to the dimension of the 


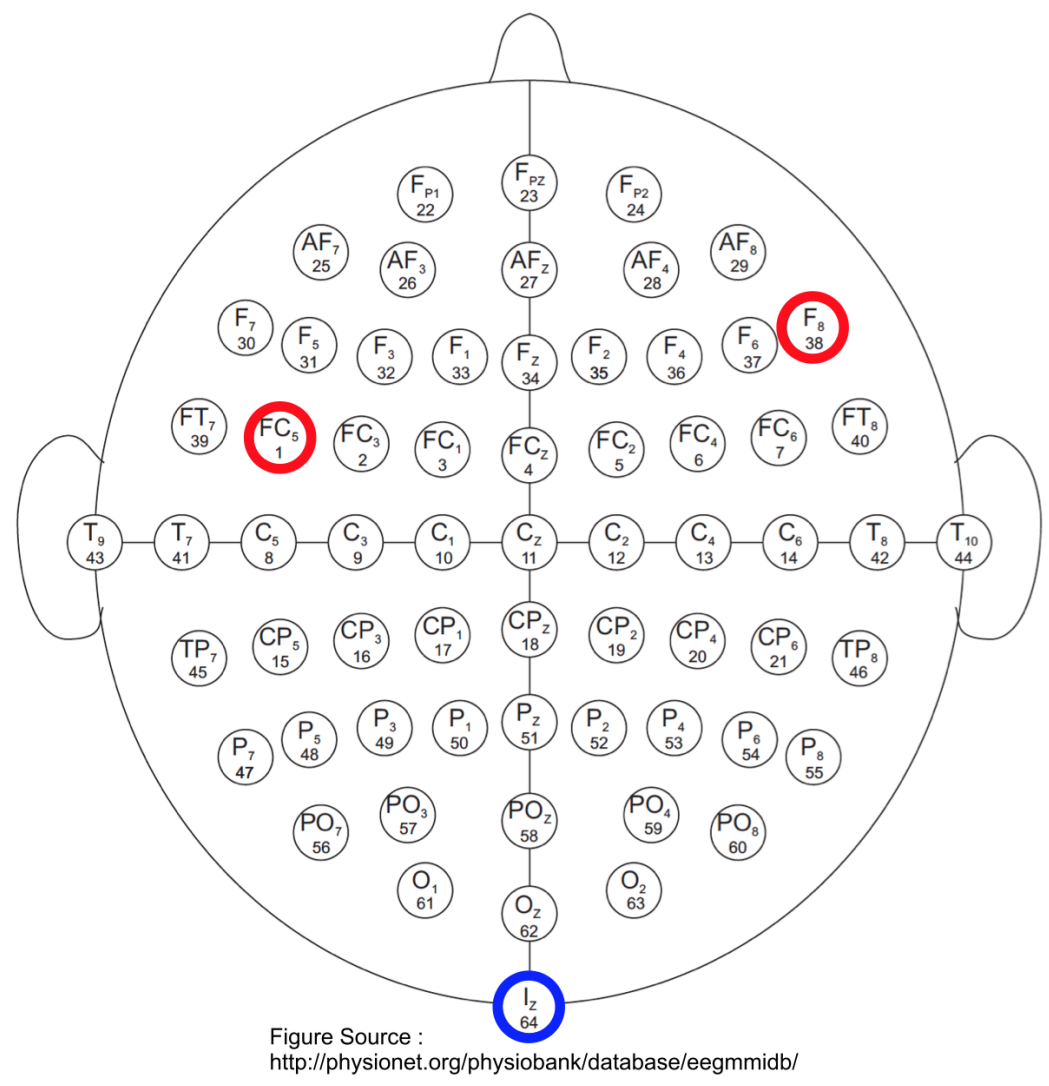

Fig. 2. Sensor distribution in the 64-channel geodesic sensor net for an EEG depicted in Figure 1 The sensor in blue represents the sensor whose neuronal activity is simulated using the fractional order system identified, and compared with data recorded in Figure 3 The sensors in red represent the minimum number of sensors required to ensure structural observability of a fractional order system identified, and considering a sparsity of $80 \%$.

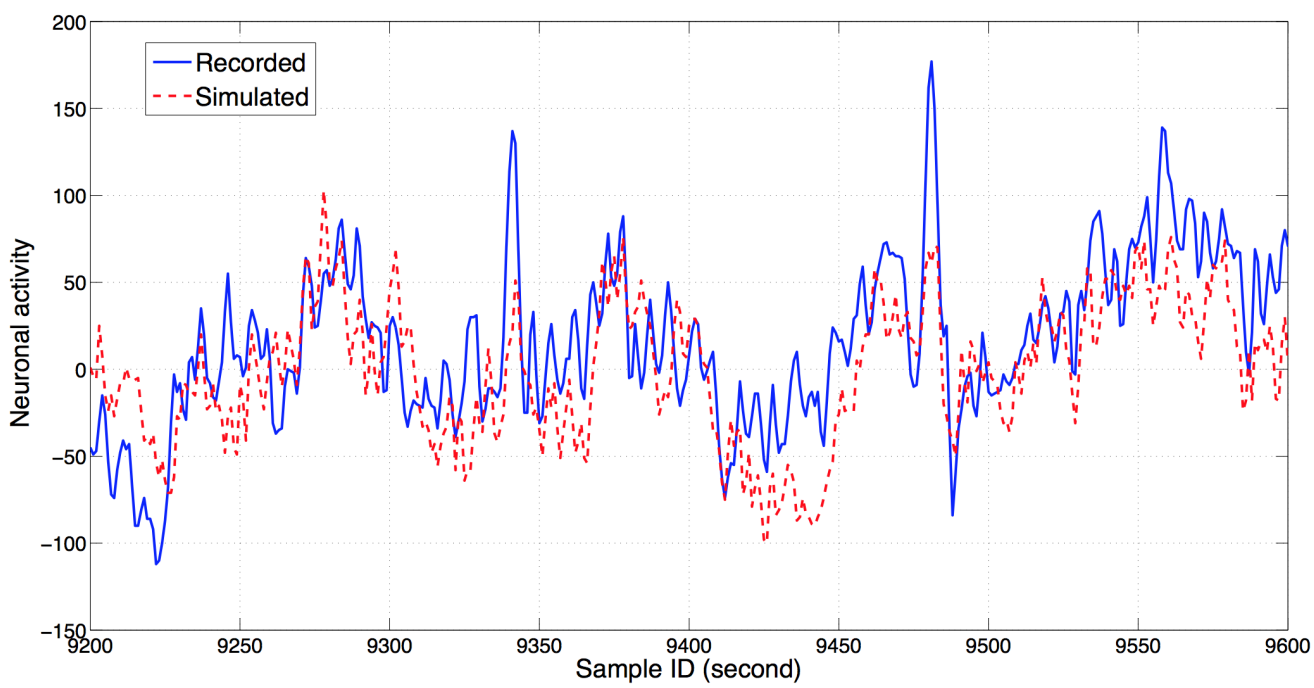

Fig. 3. In this figure, we show the recorded and simulated data on channel 64 (depicted in blue in Figure 2), where a sample is taken every second, during the first two-minutes run of interacting sessions of opening/closing the corresponding left/right fist as a function of where the targets appear on screens. 


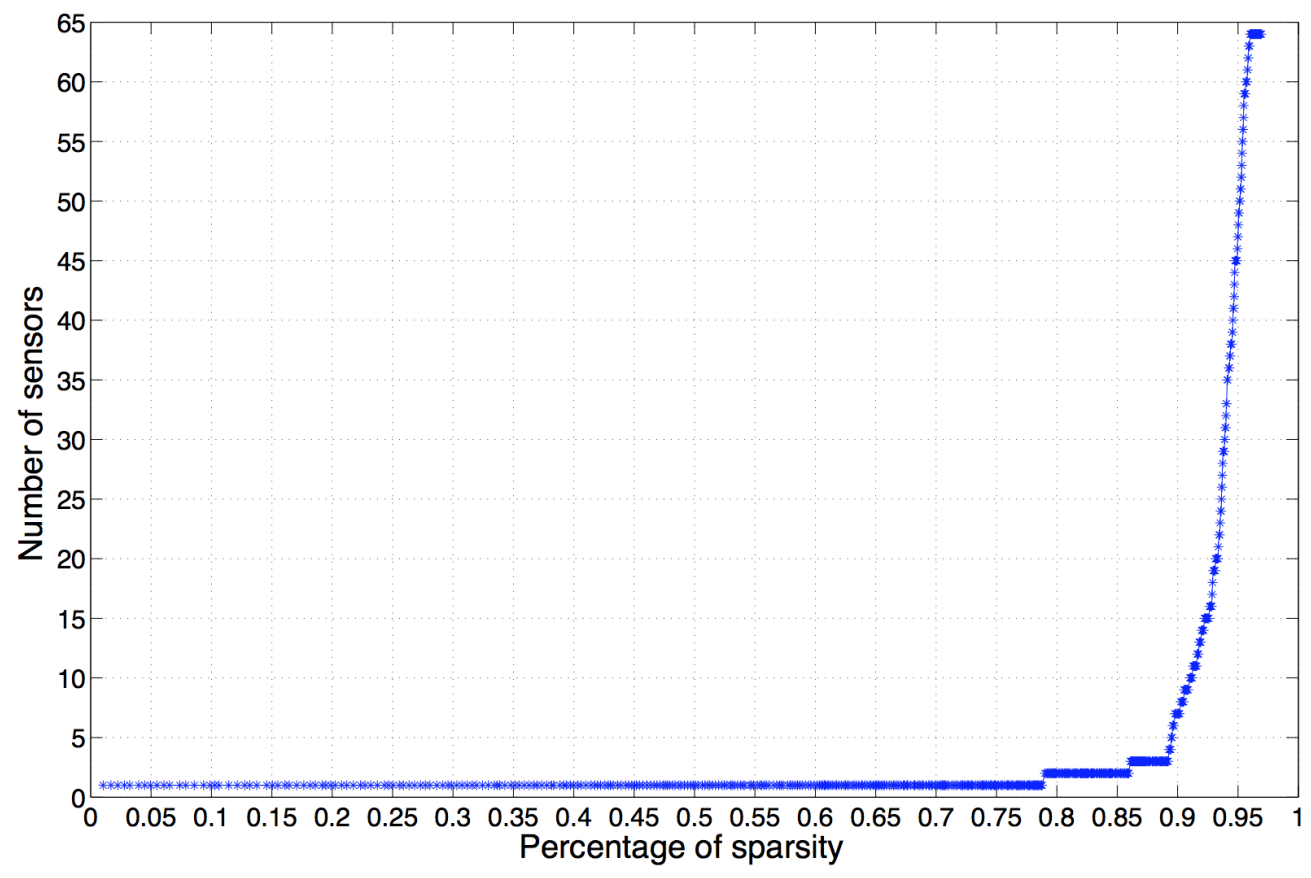

Fig. 4. Number of required sensors to ensure structural observability as a function of the sparsity.

state space. Figure 4 shows that the higher the sparsity of the coupling matrix $A$ of the brain dynamics is, the more sensors are required for observability purposes. An important observation one could make is that for the case when the system connectivity is within the range of $20-30 \%$ (which implies that the sparsity is within $70-80 \%$ interval), which is characteristic to structural/functional brain connectivity [33], [34], [35], the required number of sensors exhibit a first order phase transition. Simply speaking, to monitor the brain activity when the connectivity is between $70 \%$ and $80 \%$ we only need 2 sensors, depicted in red in Figure 2. However, when the connectivity is just $10 \%$ we are required to use 7 sensors and the required number of sensors spikes fast with increasing sparsity.

\section{CONCLUSIONS AND FURTHER RESEARCH}

In this paper, motivated by the problem of minimum placement of probes in the brain to obtain dynamic observability, we presented methodologies to determine the minimum dedicated sensor placement for discrete time fractional-order systems. Further, the results presented can be used to determine the minimum dedicated input (i.e., inputs that actuate single state variables) placement for aforementioned systems by invoking the duality between controllability and observability in the case of discrete-time fractional-order systems. Finally, it would be interesting to generalize the present results for a larger class of systems, and validate the proposed methodology in different brain regions and using different technologies.

\section{REFERENCES}

[1] Y. Tang, H. Gao, W. Zou, and J. Kurths, "Identifying controlling nodes in neuronal networks in different scales," PLoS ONE, vol. 7, no. 7, p. e41375, 072012. 
[2] S. Gu, F. Pasqualetti, M. Cieslak, S. T. Grafton, and D. S. Bassett, "Controllability of Brain Networks," ArXiv e-prints, Jun. 2014.

[3] A. Franci, G. Drion, and R. Sepulchre, "Modeling the modulation of neuronal bursting: a singularity theory approach," ArXiv e-prints, May 2013.

[4] A. Franci, G. Drion, and R. Sepulchre, "An organizing center in a planar model of neuronal excitability," SIAM Journal on Applied Dynamical Systems, vol. 11, no. 4, pp. 1698-1722, 2012.

[5] J.-S. Li, I. Dasanayake, and J. Ruths, "Control and synchronization of neuron ensembles," IEEE Transactions on Automatic Control, vol. 58, no. 8, pp. 1919-1930, 2013.

[6] A. Franci, G. Drion, V. Seutin, and R. Sepulchre, "A Balance Equation Determines a Switch in Neuronal Excitability," Mar. 2013. [Online]. Available: http://arxiv.org/abs/1209.6445

[7] G. Drion, A. Franci, V. Seutin, and R. Sepulchre, "A novel phase portrait for neuronal excitability," PLoS ONE, vol. 7, no. 8, p. e41806, 082012 .

[8] B. N. Lundstrom, M. H. Higgs, W. J. Spain, and A. L. Fairhall, "Fractional differentiation by neocortical pyramidal neurons," Nature neuroscience, vol. 11, no. 11, pp. 1335-1342, 112008.

[9] M. Ghorbani and P. Bogdan, "A cyber-physical system approach to artificial pancreas design," in Proceedings of the Ninth IEEE/ACM/IFIP International Conference on Hardware/Software Codesign and System Synthesis, ser. CODES+ISSS '13. Piscataway, NJ, USA: IEEE Press, 2013, pp. 17:1-17:10.

[10] P. Bogdan, R. Marculescu, S. Jain, and R. Gavila, "An optimal control approach to power management for multi-voltage and frequency islands multiprocessor platforms under highly variable workloads," in Sixth IEEE/ACM International Symposium on Networks on Chip (NoCS), May 2012, pp. 35-42.

[11] P. Bogdan and R. Marculescu, "A fractional calculus approach to modeling fractal dynamic games," in 50th IEEE Conference on Decision and Control and European Control Conference, Dec 2011, pp. 255-260.

[12] S. Guermah, S. Djennoune, and M. Bettayeb, "Controllability and observability of linear discrete-time fractional-order systems." Applied Mathematics and Computer Science, vol. 18, no. 2, pp. 213-222, 2008.

[13] E. Kaslik and S. Sivasundaram, "Dynamics of fractional-order neural networks," in The 2011 International Joint Conference on Neural Networks (IJCNN), July 2011, pp. 611-618.

[14] S. Zhou, H. Li, and Z. Zhu, "Chaos control and synchronization in a fractional neuron network system," Chaos, Solitons \& Fractals, vol. 36, no. 4, pp. $973-984,2008$

[15] T. Denison, M. Morris, and F. Sun, "Building a bionic nervous system," Spectrum, IEEE, vol. 52, no. 2, pp. 32-39, February 2015.

[16] S. Pequito, S. Kar, and A. Aguiar, "A framework for structural input/output and control configuration selection of large-scale systems," Accepted to IEEE Transactions on Automatic Control, 2013. [Online]. Available: http://arxiv.org/pdf/1309.5868

[17] H. Hihi, "Structural controllability of switching linear systems," Journal of Computers, vol. 4, no. 12, 2009.

[18] X. Liu, H. Lin, and B. M. Chen, "Structural controllability of switched linear systems," Automatica, vol. 49, no. 12, pp. 3531 - 3537, 2013.

[19] G. Ramos, S. Pequito, A. P. Aguiar, J. Ramos, and S. Kar, "A model checking framework for linear time invariant switching systems using structural systems analysis," in Proc. of 51th Annual Allerton Conference on Communication, Control, and Computing. IEEE Press, 2013.

[20] S. Pequito, S. Kar, and A. Aguiar, "A structured systems approach for optimal actuator-sensor placement in linear time-invariant systems," in Proc. of the 2013 American Control Conference, pp. 6108-6113.

[21] D. Baleanu, J. Machado, and A. Luo, Fractional Dynamics and Control. Springer, 2011.

[22] J.-M. Dion, C. Commault, and J. V. der Woude, "Generic properties and control of linear structured systems: a survey." Automatica, pp. 1125-1144, 2003.

[23] M. Bettayeb, S. Djennoune, S. Guermah, and M. Ghanes, "Structural properties of linear discrete-time fractional-order systems," Proceedings of the 17th World Congress of the International Federation of Automatic Control, Seoul, Korea, pp. 15262-15266, 2008. 
[24] J.-M. Dion, C. Commault, and J. V. der Woude, "Characterization of generic properties of linear structured systems for efficient computations," Kybernetika, vol. 38, pp. 503-520, 2002.

[25] C. Lin, "Structural controllability," IEEE Transactions on Automatic Control, no. 3, pp. 201-208, 1974.

[26] D. D. Siljak, Large-Scale Dynamic Systems: Stability and Structure. Dover Publications, 2007.

[27] K. J. Reinschke, Multivariable control: a graph theoretic approach, ser. Lect. Notes in Control and Information Sciences. SpringerVerlag, 1988, vol. 108.

[28] K. Murota, Matrices and Matroids for Systems Analysis, 1st ed. Springer Publishing Company, Incorporated, 2009.

[29] T. H. Cormen, C. Stein, R. L. Rivest, and C. E. Leiserson, Introduction to Algorithms, 2nd ed. McGraw-Hill Higher Education, 2001.

[30] L. A. N. Goldberger, A. L. Amaral, L. Glass, J. M. Hausdorff, P. C. Ivanov, R. Mark, J. Mietus, G. Moody, C.-K. Peng, and H. E. Stanley, "Physiobank, physiotoolkit, and physionet: Components of a new research resource for complex physiologic signals," Circulation, vol. 101, pp. e215-e220, 2000. [Online]. Available: http://circ.ahajournals.org/cgi/content/full/101/23/e215

[31] S. Achard, D. S. Bassett, A. Meyer-Lindenberg, and E. Bullmore, "Fractal connectivity of long-memory networks," Phys. Rev. E, vol. 77, p. 036104, Mar 2008.

[32] D. S. Bassett and M.-E. Lynall, "Network methods to characterize brain structure and function," Cognitive Neurosciences: The Biology of the Mind (Fifth Edition), 2009.

[33] V. K. Jirsa and A. McIntosh, Eds., Handbook of Brain Connectivity, 1st ed., ser. Understanding Somplex Systems. New York: Springer Berlin Heidelberg, December 2007.

[34] O. Sporns, Networks of the Brain, 1st ed. The MIT Press, 2010.

[35] — "Contributions and challenges for network models in cognitive neuroscience," Nature neuroscience, vol. 17, pp. 652-660, 2014. 